\title{
1 Linear vector models of time perception account for saccade and stimulus 2 novelty interactions.
}

\section{Short title: Vector models explain saccadic time distortions}

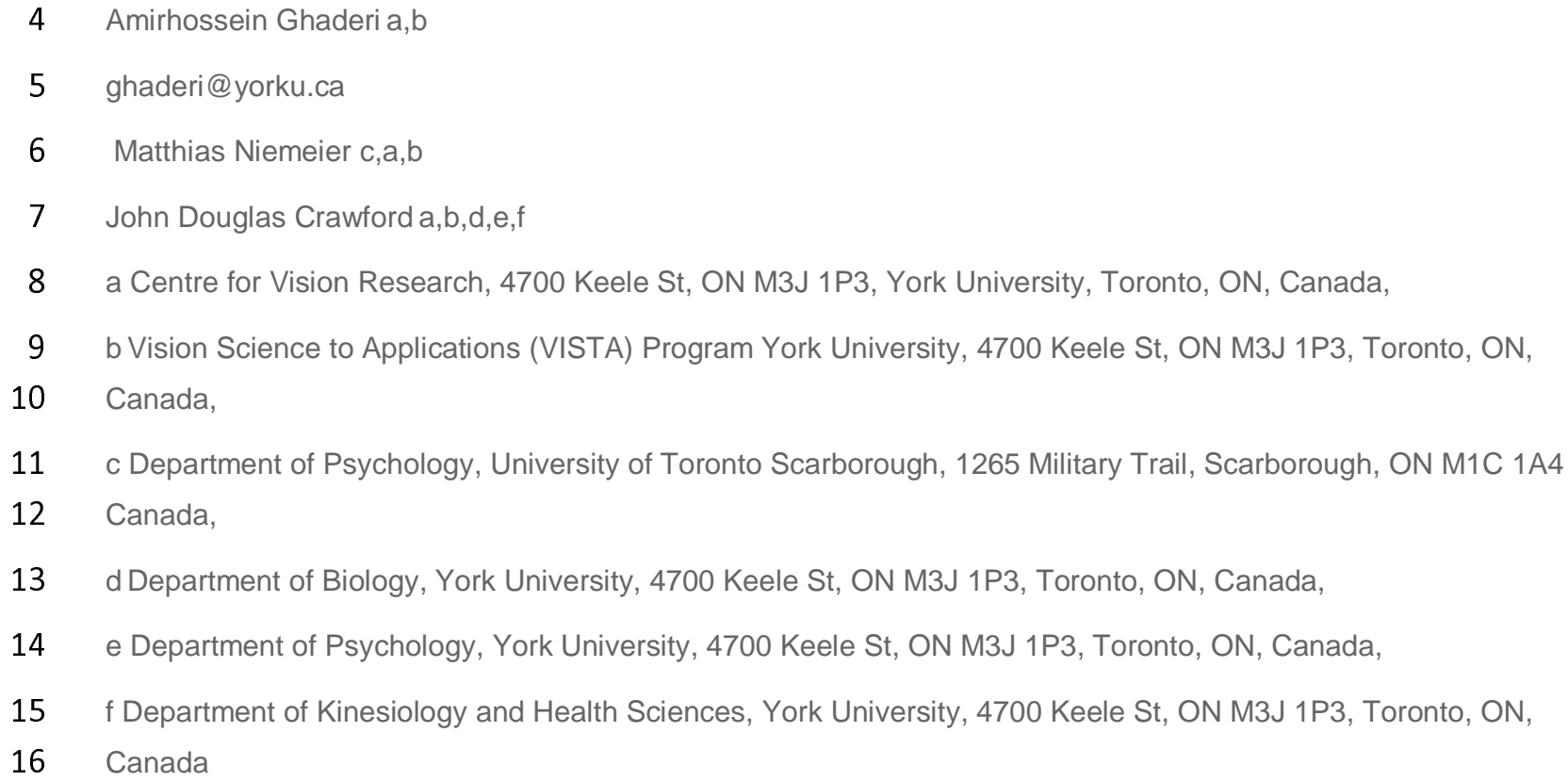

18 We thank Drs. P. Cavanagh, V. Bharmauria and P.A. Khoozani for editorial comments on the 19 manuscript, and Dr X. Yan, S. Sun, and G. Tomou for technical support. This project was funded 20 by a Natural Sciences and Engineering Research Council of Canada (NSERC) Discovery grant. 21 A.H. Ghaderi was supported by the Vision: Science to Applications Program, funded in part by 22 the Canada First Research Excellence Fund. J.D. Crawford was supported by a Canada Research 23 Chair. 
Abstract:

27 Various models (e.g., scalar, state-dependent network, and vector models) have been proposed to explain the global aspects of time perception, but they have not been tested against specific visual phenomena like perisaccadic time compression and novel stimulus time dilation. Here, in two separate experiments $(\mathrm{N}=31)$, we tested how the perceived duration of a novel stimulus is influenced by 1) a simultaneous saccade, in combination with 2) a prior series of repeated stimuli in human participants. This yielded a novel behavioral interaction: pre-saccadic stimulus repetition neutralizes perisaccadic time compression. We then tested these results against simulations of the above models. Our data yielded low correlations against scalar model simulations, high but non-specific correlations for our feedforward neural network, and correlations that were both high and specific for a vector model based on identity of objective and subjective time. These results demonstrate the power of global time perception models in explaining disparate empirical phenomena and suggest that subjective time has a similar essence to time's physical vector.

Keywords: time perception, perisaccadic time distortion, stimulus repetition, vector timing, neural network, scalar timing

\section{1- Introduction}

Time perception studies generally assume that subjective or perceived time is distinct from objective time(1-3) and have explained this difference from two opposing perspectives: either from the perspective of global theories of time perception, or post-hoc explanations of specific empirical phenomena. Previous theories of time perception include linear dedicated models(4) such as internal clock models $(2,3,5)$, and non-linear models such as state-dependent networks (SDNs)(6-8). Conversely, empirical studies have shown that various sensory and behavioral factors influence human time perception(9-13), for example, perisaccadic time compression (12-18), and dilated time for a novel visual stimulus following repeated stimuli(19-21). 
opposed to post-saccadic time expansion(22)) has been explained in terms of perisaccadic remapping $(14,15,17)$ and transient cortical responses $(23)$, whereas temporal expansion of a novel stimulus (after a series of repeated stimuli) has been attributed to a release from repetition suppression mechanisms $(20,21,24)$. However, there is also a need to bridge these two levels of explanation (global theories vs. mechanistic models based on empirical data), because the high level models need to be empirically tested, and the low-level explanations might benefit from a unifying theory $(25,26)$.

Here, we attempted to reconcile these approaches by 1) using empirical data (specifically, the previously unexplored interactions between perisaccadic time compression and time dilation of a novel stimulus after a series of repeated stimuli), 2) using these data to test between the predictions of the three major 'high level' models mentioned above (and explained below), and then 3) interpreting our findings with regard to 'low level' mechanistic descriptions of our findings.

In scalar timing models (Figure 1-a, Table 1), subjective time is viewed as a scalar parameter that can be generated by an internal pacemaker/accumulator structure(3) or related to levels of energy spent in the brain $(13,27)$. Such models predict that specific time distortion effects (e.g., stimulus repetition vs. saccades) should add linearly. In contrast, SDN models $(6-8,28)$ (Figure 1b, Table 1) are nonlinear(8), being based on classification network training. Specifically, these models assume that time distortion effects can be coded by different connectivity patterns in specific neural networks(29). Such models can account for possible non-linear interactions between stimulus repetition and saccades. Finally, we considered a recently proposed vector model for subjective time (Figure 1-c, Table 1), based on a physical concept of time $(30,31)$. In this model, neural time units are defined by a directional arrow and a magnitude (i.e., a vector)

75 as in temporal physics(31-33). In this framework, simultaneous time distortions can be integrated by a neuro-information approach $(30,31)$. For example, if the distortion effects of

77 saccade and stimulus repetition are added to a vector of subjective time, they can then expand or compress subjective time within this vector space. 
79 To evaluate these models, we employed a repetitive stimulus / saccade / novel stimulus paradigm in human participants (Figure 2). In two separate experiments (with different stimulus durations and setups), participants judged the duration of a novel test stimulus relative to a previously viewed series of reference stimuli with a different orientation (Figure 2-a). After the reference stimuli, we cued a saccade, which commenced toward the end of the test stimulus (Figure 2-b. We then analyzed the interactions between perisaccadic time compression and repetition-induced time dilation of the test stimulus and compared these to simulations of the models described above. This yielded the novel results that time dilation after a series of repeated stimuli supersedes saccade-induced time compression, and more importantly, linear vector model simulations provided the best fits to this data (in terms of accuracy and specificity) compared to the other models of time perception. This demonstrates that global time perception models can explain specific empirical phenomena and suggests that subjective time follows the rules of objective time in a physical framework.

\section{2- Method}

To generalize our psychophysical results, and obtain sufficient data for model testing, we include data here from 31 participants tested in two separate experiments.

\section{2-1 Experiment 1}

2-1-1 Participants. Ten volunteers (age: mean=31.5, range between 23 and 39, 5 female) participated in the first experiment (including one of the authors; A.G.). Further data collection in this first experiment was interrupted by the pandemic. All the participants had normal/corrected to normal vision and they did not report any visual or movement disorder. All the participants signed a written informed consent. The details of experimental procedures, data collecting, and saving were described in this informed consent. This study (including the materials of informed consent) was approved by the office of research ethics (ORE) at affiliated universities. All methods in this study were performed in accordance with the Declaration of Helsinki. 
106

107

108

109

110

111

112

113

114

115

116

117

118

119

120

121

122

123

124

125

126

127

128

129

130

131

132

133

2-1-2 Setup. Participants sat in a dark room with their head fixed with a dental impression bar. Eye movement tracking was performed (in the Saccade and Fixation Tasks) using an Eyelink-2 system via a camera that was focused on the right pupil. Forty centimeters in front of the eyes a black display screen $\left(1.9 \times 1.4 \mathrm{~m}\right.$; luminance level of $0.015 \mathrm{~cd} / \mathrm{m}^{2}$; temporal resolution: $\left.60 \mathrm{~Hz}\right)$ presented rear-projected stimuli that were programed in $\mathrm{C}++$ and build by Borland $\mathrm{C}++$ version 5.02 .

2-1-3 Stimuli. Tree white parallel horizontal lines (thickness of line: $1^{\circ}$, distance between them: $4^{\circ}$, total size $11^{\circ} \times 11^{\circ}$ ) were presented as reference stimulus, while the test stimulus had the same configuration but with different orientation (vertical lines). Stimuli were presented in the right visual field $\left(10^{\circ}\right.$ from the center) and a fixation point was horizontally shown at center $(x=0)$. In the Fixation Task, a fixation point was randomly presented at $12.5^{\circ}$ above or below of center $\left(x=0^{\circ}, y= \pm 12.5^{\circ}\right)$, while the location of stimuli was $x=10^{\circ}$ and $y=0^{\circ}$. In the Retina-Matched Fixation Task, the fixation point was always presented at center $\left(x=0^{\circ}, y=0^{\circ}\right)$ but stimuli (both of reference and test) were randomly shown $12.5^{\circ}$ above or below of center line $\left(x=10^{\circ}\right.$, $\left.\mathrm{y}= \pm 12.5^{\circ}\right)$. In the Saccade Task, the location of stimuli was same as the Fixation Task $\left(x=10^{\circ}\right.$, $y=0^{\circ}$ ) but the location of the fixation point was randomly changed from $x=0$ and $y= \pm 12.5$ to a symmetric location below or above an imaginary horizontal line passing through the vertical center of the screen. All the stimuli were shown on a black background.

2-1-4 Trials details and experiment design. The experimental design was simplified and optimized to provide statistical power for testing the models described in subsequent Methods sections. In each task, 240 trials were presented. There were two conditions according to repetition of reference stimulus: No repetition and repetition (randomly 1 or 2 times). Duration of test stimulus was $140 \mathrm{~ms}, 170 \mathrm{~ms}, 230 \mathrm{~ms}$, or $260 \mathrm{~ms}$ in different trials. By comparing these durations with the duration of the reference stimulus, trials were divided into longer (test duration longer than reference) and shorter trials (test duration shorter than reference). Then, in each task, 60 trials $(240 /(2 * 2))$ are allocated to each condition. These conditions were presented in three different tasks: Saccade task, Fixation task, and Retina-matched task. In all the tasks, a reference stimulus preceded a test stimulus. Duration of the reference stimulus was 
always $200 \mathrm{~ms}$ and interstimulus interval was $300 \mathrm{~ms}$. In the Saccade Task, the fixation point was moved $100 \mathrm{~ms}$ before the presentation of the test stimulus $(200 \mathrm{~ms}$ after reference stimulus presentation). After presentation of the test stimulus participants were asked to judge which stimulus was longer, test or last reference stimulus. The procedure is illustrated in Figure 2. Trials counterbalanced location of fixation point and stimuli (i.e., in 120 trials the test stimulus was shown above and in 120 trials it was shown below the middle of the screen).

2-1-5 Saccade latencies. Saccades were detected by Eyelink-2 system and recorded by a subroutine generated by visual $\mathrm{C}++$ in two separate computers. The eye position signals were sent via serial port from the eye tracking computer (first device) a recording computer (second device). The task was presented on a different computer (third device) and a trigger was sent via parallel port to eye movement recording computer (second device) when the location of fixation point was changed (saccade command). The time difference between saccade command and eye movement was considered as saccade latency. The acceptable saccade latency was between $150 \mathrm{~ms}$ and $400 \mathrm{~ms}$. Trials with longer or shorter latencies were removed. Since the test stimulus was always presented during saccade preparation / execution (perisaccadic interval) and vanished before fixation of the eyes at their new position (Figure 2b), the related time distortion effect was considered to reflect perisaccadic time compression(14) rather than time expansion or chronostasis which may occur immediately following a saccade(22).

\section{2-1-6 Statistical analysis.}

Percentage of incorrect responses for longer and shorter trials were calculated in each condition. Incorrect responses for longer trials show underestimation of time while percentage of incorrect responses of shorter trials exhibit overestimation. We performed permutation $\mathrm{t}$ tests with 5000 random shuffling for each type of these trials to evaluate the main effects of tasks and repetition. With three tasks and repetition/no-repetition of reference stimulus combinations, we had a total of 6 conditions, i.e., Saccade/No-repetition (S-NoRep), Saccade/Repetition (S-Rep), Fixation/No-repetition (Fix1-NoRep), Fixation/Repetition (Fix1Rep), and Retina-matched Fixation/No-repetition (Fix2-NoRep), Retina-matched 
162 Fixation/Repetition (Fix2-Rep). False discovery rate (FDR) was performed to correct for multiple comparison errors. We also subtracted repetition effects from tasks to evaluate interactions between them. To perform this analysis, we compared three pairs: ( $S$-Rep minus S-NoRep) and (Fix1-Rep minus Fix1-NoRep), (S-Rep minus S-NoRep) and (Fix2-Rep minus Fix2-NoRep), (Fix1Rep minus Fix1-NoRep) and (Fix2-Rep minus Fix2-NoRep). Post hoc analysis, using extra permutation t-tests, were also performed among different tasks in repetition condition (i.e., SRep, Fix1-Rep, and Fix2-Rep). Statistical analysis was performed in MATLAB R2019a.

\section{2-2: Experiment 2}

2-2-1 Participants. 21 volunteers (age between 18 and 44, 12 female) participated in the second experiment. All participants were different from the first study. The inclusion/exclusion and ethical criteria were similar to the first experiment, except the experiment was approved by both affiliated universities.

2-2-2 Setup. Stimuli were presented on a 25 " LED screen (refresh rate: $144 \mathrm{~Hz}$, full HD). Similar to the first experiment, participants sat in a dark room while their distance from the screen was $35 \mathrm{~cm}$. Fast eye movements tracking was performed (in the Saccade and Fixation Tasks) by recording electrooculography (EOG) at $2048 \mathrm{~Hz}$ using $\mathrm{Ag} / \mathrm{AgCl}$ electrodes (located at both sides of eyes) and a Biosemi amplifier. The task was programed and run using Psychtoolbox-3 (version 3.0.16) in MATLAB R2019a. Note that these data were recorded in conjunction with EEG data that are not relevant, and therefore not described or included, in the current paper.

2-2-3 Stimuli. Shape and size of stimuli and fixation point were similar to the first experiment. But in the saccade task fixation point was moved horizontally from $x=0, y=0$ randomly to $x= \pm 12.5, y=0$ (rather than vertically as in the first experiment).

2-2-4 Trials details and experiment design. Participants performed a saccade and a fixation task in two different sessions (the second session was conducted one week after the first). In total 720 trials were collected in each task. In this experiment, presentation of stimuli was similar to the first experiment, except timing was changed. We set the duration of the test stimulus to $70 \mathrm{~ms}$ and the duration of the reference to $30,50,90$, or $110 \mathrm{~ms}$. The interstimulus 
interval was $300 \mathrm{~ms}$. After presentation of the test stimulus participants judged which stimulus was longer, test or last reference stimulus.

\section{2-2-5 statistical analysis:}

As in the first experiment, percentage of incorrect responses for longer/shorter trials were obtained in each condition to evaluate underestimation/overestimation of time. Permutation ttests with 5000 random shuffling for each type of trials were performed and the main effects of tasks and repetition were compared. In this experiment, two tasks were presented, and we had a total of 4 conditions, i.e., Saccade/No-repetition (S-NoRep), Saccade/Repetition (S-Rep), Fixation/No-repetition (F-NoRep), and Fixation/Repetition (F-Rep). We performed false discovery rate (FDR) to correct for multiple comparison errors. Furthermore, we subtracted repetition effects from tasks to evaluate interactions between them comparing two pairs: ( $S$ Rep minus S-NoRep) and (F-Rep minus F-NoRep). We also performed extra permutation t-tests as post hoc analysis to compare different tasks in the repetition condition (i.e., S-Rep and FRep). Statistical analysis was performed in MATLAB R2019a.

2-5 Exclusion criteria. The exclusion criteria for rejected trials were as follows: 1) Trials with saccades during standard/test stimuli presentations during fixation intervals. 2) Saccade trials with late (later than 400 ms after fixation-point movement) or early (earlier than 150ms after fixation-point movement) saccade execution. 3) Trials with no responses. 4) Trials where participants were affected by any unintended external stimulus (e.g., extra noises, talking, movement etc.) during the trial presentation. Based on these criteria, the percentage of data exclusion was less than $30 \%$ of all trials for each participant (mean=16.8\%, SD=8.79\%). Trial rejection was performed by self-developed subroutines in MATLAB R2019a.

\section{2-6 Models.}

2-6-1 Preparing data for models. We calculated individual timing performance (ITP) as a person's ability to perceive time in a given condition relative to their ability in the control conditions (i.e., conditions with the minimum of distortion effects). To this end, we first obtained the percentage of trials that were correctly judged by each participant in each 
condition. The percentage of correctly judged trials in the conditions with no time distortion effects (i.e., repetition and saccade) was considered as pure time $\left(t_{\text {pure }}\right)$. These trials were obtained from both Fixation Tasks where the reference stimulus was presented without repetition (when there were neither repetition nor saccade effects on timing judgment) (See Figure 2-c). Equivalently, the $t_{S}, t_{R}, t_{S R}$ were defined as the percentage of trials that were correctly judged in the saccade, repetition, and saccade/repetition conditions, respectively. Next, we calculated the ratio of $t_{S}, t_{R}, t_{S R}$ in comparison to $t_{p u r e}$ and the ITP for each condition was defined as:

$$
\left.I T P_{S, R, S R}=\frac{t_{S, R, S R}}{t_{\text {Pure }}} \quad \text { (eq. } 1\right)
$$

The ITPS were calculated for both types of trials (i.e., trials with shorter or longer duration of test stimulus in comparison to the duration of reference stimulus). ITP $=1$ shows the duration judgment in presence of time distortion effects is similar to the duration judgment in the conditions without distortion effects. ITPS $>1$ signify the time distortion effects increased accuracy of test stimulus duration judgment, whereas ITPS $<1$ signify the distortion effects reduced accuracy to judge to duration of test stimulus relative to the reference stimulus. ITPS were calculated for each participant using MATLAB R2018a.

Then, we tested three different models (linear scalar model, linear vector model and state dependent network model) based on previous hypotheses about time perception (Figure 1, 3). In all three models, the same two inputs $I T P_{R}, I T P_{S}$, and same output $I T P_{S R}$ were utilized. Three criteria were selected to test the models. 1) Sensitivity (outputs of the model should correlate with the actual behavioral data). 2) Specificity (i.e., for specific model predictions these correlations should disappear when the data are randomly shuffled, whereas correlations due to overfitting the data should persist). 3) Generality (models should be compatible with different experimental conditions and various type of time judgments). These criteria were determined through linear correlation analysis and calculating root mean square error (RMSE) between output of models and original/shuffled data. 
2-6-2 Description of models. The linear scalar model was implemented based on a wide range of studies that considered the internal clock and scalar expectancy theory $(3,34)$. In this model, saccade and repetition have been considered as distortion effects that can accelerate or decelerate the accumulation rate of pacemakers (clocks). This acceleration/deceleration can be multiplied to perceive time in absence of distortions $\left(t_{P \text { ure }}\right)$ by applying positive coefficients to adjust the rate of pacemakers (coefficients are higher than 1 for accurate time estimation and lower than 1 for inaccurate estimation). All the possible coefficients were applied to find the best performance of the model. A schematic/mathematic framework of this model has been presented in Figure 3.

251 The second model was implemented based on state dependent network models (6-8). To this end, a feedforward artificial neural network (ANN) with multi layer perceptron structure (two inputs perceptron, one hidden layer with 100 perceptron units in hidden layer and one output perceptron) was employed. A back-propagation algorithm was used for ANN training. We used $85 \%$ of dataset as training data and $15 \%$ of the dataset as test data. To evaluate the effect of different datasets on efficacy of ANN and to avoid overfitting, we used Bayesian regularization backpropagation algorithm that is a robust algorithm against overfitting problem. We consider the results of training and testing by looking at correlation value ( $R$ values) and RMSE between fitted values (by ANN model) and original values (from behavioral experiment). The mathematical processes of this modeling method are presented in Figure 3. We used artificial neural network toolbox in MATLAB 2019b to create the ANN model.

The third model was accomplished based on our previous hypothesis that state time in the brain has the same properties as physical time, represented by via vector units $(30,31)$. In this model, time distortions are considered as different vector units to strengthen (dilatate) or destroy (compress) the vector of perceived time, or these effects can cancel in the absence of distortion effects $\left(t_{\text {Pure }}\right)$. This linear vector model was implemented by applying the $\cos (\phi, \psi)$

267 (instead of the acceleration/deceleration weights of perceived time in scalar models). Where $268 \phi$, and $\psi$ were the assumed angles between the vectors of time distortion effects (i.e., saccade and repetition, respectively) and the veridical vector of time in the absence of time distortion 
effects. This model follows a linear formula where two distortion effects are linearly added to $t_{\text {Pure }}$. All the possible angles were tested, and the performance of model was obtained for these various angles. Figure 3 depicts this model mathematically and schematically. All the models have been generated by MATALB 2019b.

\section{3- Results}

\section{3-1 Behavioral results}

3-1-1 Experiment 1: Ten participants performed this experiment, in counterbalanced blocks corresponding to three tasks (Figure 2-a). After analyzing saccade latency, one participant was excluded according to exclusion criteria. In each task, participants judged the variable duration $(140,170,230$, or $260 \mathrm{~ms})$ of a test stimulus (a horizontal or vertical grid) relative to the fixedduration (200ms) of the last stimulus in a previously viewed series of 1 to 3 reference stimuli with the opposite (orthogonal) orientation (Figure 2-a). Participants were initially cued (by a small cross) to fixate their gaze just beyond one of four corners of the centrally located reference stimuli. In the main Saccade Task, this fixation cross shifted to the adjacent corner, triggering a vertical saccade with a stereotypical latency (268.4 $\pm 35.5 \mathrm{~ms})$. The test stimulus appeared just before saccade onset, such that saccades commenced at or near the end of the test stimulus, depending on its duration (Figure 2-b). Note that in this task, the test stimulus appeared at the same location as the reference stimuli but stimulated a different area of the retina (i.e., same spatial, different retinal location). To control for these spatial factors, we also included a Fixation Task, where the test stimulus appeared at the same location, but the fixation cross did not shift to induce a saccade (same spatial, same retinal location), and a Retina-Matched Fixation Task, where the test stimulus shifted like the fixation cross in the Saccade Task to stimulate the retinal location of the pre-saccadic stimuli (different spatial, same retinal). Thus, comparisons were performed among 6 conditions, i.e., Saccade/No-repetition (S-NoRep), Saccade/Repetition (S-Rep), Fixation/No-repetition (Fix1-NoRep), Fixation/Repetition (Fix1-Rep), and Retina-matched Fixation/No-repetition (Fix2-NoRep), Retina-matched Fixation/Repetition (Fix2-Rep). Furthermore, interaction of repetition and task was evaluated. 
298 To help distinguish the effects of saccades (time underestimation) and repetition 299 (overestimation) we divided the data into incorrect response for shorter versus longer trials.

300 Figure 4-a shows the percentage of incorrect responses for shorter trials (overestimation) in 301 different conditions (individual responses were presented by color dots and distributions of 302 responses were presented by violin plots). FDR revealed significant differences between 303 repetition and no-repetition conditions in all tasks. This suggests repetition causes 304 overestimation of time either in fixation or saccade tasks. No significant interaction was 305 observed between repetition effect and tasks. Statistical details are presented in the caption of 306 figure 4.

307 Incorrect responses for longer trials (underestimation) are presented in figure 4-b. In this figure 308 individual responses were presented by color dots and distribution of responses were 309 presented by violin plots. FDR revealed significant decreased underestimation in repetition conditions in comparison to repetition conditions in all tasks. Comparisons between tasks revealed significant time underestimation in the saccade condition (in comparison to both 312 fixation conditions). No significant differences were observed between two eye-fixed 313 conditions. Furthermore, we observed significant interactions between repetition effects and 314 tasks (S-Rep minus S-NoRep) vs. (Fix1-Rep minus Fix1-NoRep) (t=-3.12, $\mathrm{p}=0.014)$ and (S-Rep 315 minus S-NoRep) vs. (Fix2-Rep minus Fix2-NoRep) $(\mathrm{t}=-5.06, \mathrm{p}<0.001)$ ). Post hoc analysis revealed 316 that there is no significant difference between S-Rep, Fix1-Rep, Fix2-Rep conditions. It suggests 317 that repetition cancels the underestimation effect of saccades. Statistical details are presented 318 in the caption of figure 4.

319 These results show that overestimation of time was always present after repetition of the 320 reference stimulus, and that this effect superseded the time compression effect in the presence 321 of a saccade. The underestimation of time for individual participants was always observed in 322 the saccade-norepetition condition whereas when the reference stimulus was repeated no 323 significant underestimation was observed in the saccade task.

324 3-1-2: Experiment 2: 21 participants performed this experiment. Three participants were 325 excluded because of exclusion criteria (inappropriate saccade latency). This experiment was 
similar to the first experiment, except two tasks (fixation and saccade) (instead of three tasks) were performed by participants and timing was different (duration of test was 70ms and reference stimulus duration was one of the variable durations: $30,50,90,110 \mathrm{~ms}$ ). Furthermore, participants performed horizontal saccades instead of vertical. In the Saccade Task, latency of saccade was $263.7 \pm 41.4 \mathrm{~ms}$.

Figure 5-a presents the percentage of incorrect responses for shorter trials (overestimation of time) in different conditions (individual responses were presented by color dots and distributions of responses were presented by violin plots). The FDR analysis revealed that repetition significantly increased incorrect responses in both tasks that means pre-stimulus repetition cause to overestimation of time either in fixation or saccade tasks. No significant difference was observed between fixation and saccade tasks. Furthermore, interaction between repetition effect and tasks was not significant. Statistical details are presented in the caption of figure 5 .

Figure 5-b shows incorrect responses for longer trials (underestimation of time). In this figure, individual responses were identified by color dots and distribution of responses were presented by violin plots. FDR revealed significant time underestimation in the saccade/no-repetition condition in comparison to fixation/no-repetition condition. Significant differences were also observed between repetition and no-repetition conditions in both tasks (i.e., S-NoRep vs. S-Reo and F-NoRep vs. F-Rep). Significant interaction was observed for repetition effect and task (t= 2.56, $p=0.021$ ). Post hoc analysis showed that there is no significant difference between SR and FR conditions. This later result suggests that repetition cancels the underestimation of time in saccade condition.

Consistently, this experiment showed that repetition of a stimulus before the test stimulus led to overestimation of perceived duration for test stimulus. Also, saccade compressed the perceived duration of test stimulus that was presented $100 \mathrm{~ms}$ before saccadic eye movement. Like the first experiment, results of the second experiment confirm that this saccadic time compression is canceled when a repeated series of stimuli is presented before the saccade.

\section{3-2 Modeling results}


354 In the second part of this study, we defined individual timing performance (ITP) by comparing the individual time judgments in trials with time distortion effects (i.e., repetitions and saccade) and trials without distortion effects. We integrated behavioral results from both experiments and used the calculated ITPs (for both experiments and longer or shorter trials) to test between the models shown in Figure 1. To test the ability of each model to explain our observed Saccade-Repetition (SR) interactions, the Saccade-NoRepetition condition (S), and FixationRepetition condition (FR) were used as inputs to the model and the outputs were responses in the Saccade-Repetition condition (SR), i.e., each model was required to integrate the separate saccade and repetition effects in order to reproduce the combined effect. Each model was trained or optimized to best fit the actual output data. Figure 6 illustrates the goodness of fit of each model against the data as scatter plots (model output as a function of actual Saccade-Repetition data), and beneath these, the corresponding residuals of fit (i.e., Euclidean distances between outputs of models and the data). The inputs are derived from $I T P_{R}$ and $I T P_{S}$ in two experiments and output for this set was ITP $P_{S R}$. In the first experiment, since we had two fixation conditions, ITP and ITP $P_{R}$ were calculated for fixation/saccade, and retina-fixed/saccade condition combinations. This ITP set were separately calculated for longer and shorter trials. Then, in this experiment, four ITP set (fixation/saccade-longer trials, fixation/saccade-shorter trials, retina-fixed/saccade-longer trials, and retina-fixed/saccade-shorter trials) were obtained for each participant (for all nine participants we had

372 totally $4 \times 9=36$ ITPs). For the second experiment, ITP set of 18 participants were calculated for 373 fixation/saccade trial combination. For each participant two ITP set were obtained according to their responses for longer and shorter trials. Then, total number of ITPs were $2 \times 18=36$ in this experiment.

375 According to the third criterion, a good model should be valid against different task conditions (e.g., 376 different durations), then we integrated all ITPs from two experiments and both longer and shorter 377 trials. Totally 72 ITP sets were used as input/output samples for models. Figure 6-a shows fits made to 378 the original data whereas the part (b) shows fits made to shuffled data (see methods) as a control. To 379 quantify the goodness of fit for each model, we performed regression analysis (Table 2) that accounted 380 for the different combinations of input used for testing and participant number as factors and took the 381 Root Mean Square Error (RMSE) of the residuals. The best model should show good fits that are specific, 382 i.e., high correlations and low residuals, and only to the original data, not the shuffle control for all 383 samples (regardless of experiment or type of trials). 
After Bonferroni correction, all three models showed significant correlations between fitted outputs and original data. high correlation values (R-values>0.7) were obtained using the vector and ANN models. The scalar model showed moderate correlation (R-value=0.64). The highest accuracy (largest R-value and smallest RMSE) was achieved using linear vector model (table 2). This showed that the vector and ANN models are reliable models to describe both distortion effects in different condition. But the scalar model is in moderate range of sensitivity. Figure 6-a showed that scalar model was particularly reliable only in one experiment.

As shown in figure 6-b and table 2, when we tested three models against random-shuffled data to examine specificity of models, two models (the scalar and vector models) showed nonsignificant correlations between fitted outputs and random-shuffled outputs. However, ANN exhibited significant moderate correlation ( $\mathrm{R}=0.57)$. This suggest that the scalar and vector models are specific models for time perception, but ANN is not a specific model.

In summary, the performance of scalar model was in moderate range, the neural network model performed better but was not specific to the actual dataset, whereas the linear vector model fit both of our criteria: it performed well and was specific to the original unshuffled datasets for both experiments.

\section{4- Discussion}

In this study, we asked how two separate time distortion effects (repetition time dilation and perisaccadic time compression) interact, and which time perception model can best explain the derived results. Overall, the behavioral results confirm previous findings on perisaccadic time compression(14,35) and relative effect of time dilation after a series of repetitive stimuli $(20,21,24)$ but expand on this by showing how they interact. Results showed the relative time dilation induced by repetition of a reference stimulus before test stimulus neutralized the presence of an intervening saccade (i.e., the interactive effect of perisaccadic time compression and repetition time dilation is similar to single effect of repetition time dilation alone). To model this interaction, the resulting data were used as inputs/outputs for models of time perception (scalar clock, non-linear artificial neural network, linear vector). The linear vector model performed best, both in terms of specificity and ability to fit the data. Here, we will 
412 consider the experimental results in more detail, and discuss their implications for broader time 413 perception theories.

414 In agreement with previous investigations(12,14,15,35), our results showed that saccades 415 compress the subjective perception of the duration of a briefly presented visual stimulus. 416 Whereas previous investigations used empty temporal intervals (i.e. the interval between 417 presentation of two stimuli(14) has been judged instead of duration of one presented stimulus), 418 we presented the test stimulus during perisaccadic interval and participants judged the 419 duration of the stimuli themselves. This demonstrates that, notwithstanding the presence of 420 different mechanisms for time perception of brief empty or filled intervals(36), saccadic time 421 compression occurs in both cases. On the other hand, our results support previous 422 studies $(13,20,21,24)$ that found the relative duration of a novel stimulus after a series of 423 repeated stimuli is perceived to be longer than the last repeated stimulus (in the repetitive 424 series) with the same objective duration.

425 In the Retina-Matched Fixation Task (first experiment), the test stimulus was presented in a 426 different spatial location (but same retinal location) relative to the repeated reference stimulus. 427 Thus, in this task, the test stimulus shows two types of novelty compared to the reference 428 stimuli: different location and different orientation. However, in this condition time dilation was 429 not significantly different from that in our Fixation Task (where the test stimulus was presented 430 in the same objective and retinal location as the reference stimulus). This suggests that 431 objective spatial location did not have a strong effect on perceived time. This might be because 432 the new location of our test stimulus was always predictable, and one could predict that the 433 test stimulus would be presented in another possible location that was different from the 434 location of reference stimulus. Whereas the randomized number of repetitions was not 435 predictable in all tasks.

436 Various, often disconnected explanations have been provided for these phenomena. Saccadic 437 remapping(14,37), transient responses in visual cortex(23), saccadic suppression $(16,27,37,38)$ 438 and attention(16) have been assumed to be associated with perisaccadic time compression, 439 whereas time dilation of a novel stimulus (after a series of repeated stimuli) has been 
explained by attention(24) and repetition suppression mechanisms $(13,27)$. The latter two explanations (neural suppression and attention) are common to both phenomena (saccadic time compression and time dilation after a series of repeated stimuli) but the other proposed mechanisms for saccadic suppression can not explain time dilation for the novel stimulus following a repeated stimulus. Therefore, when considering how these phenomena might interact, we will focus on attention and neural suppression.

We will first consider the joint effects of attention on time distortions induced by saccade and repetition $(16,24)$, because this can be directly related to the internal time clock model simulated above. In the internal clock model, attention is considered as a switch between the pacemaker and accumulator(3,5,39) (Figure 1-a). Higher levels of attention allocation can turn on this switch, thus causing the accumulator to collect more timing pulses and overestimate time $(24,39)$. Alternatively, a recent saccade / time perception study suggested the decreased attentional allocation to the stimulus leads to decreased time pulse accumulation in the internal clock, thus resulting in perisaccadic time compression(16).

Based on this model, subjective time is a scalar parameter(3) that has accumulative property. This model thus predicts that a scalar combination of saccade and repetition trials should correlate with saccade-repetition trials (Figure 1-a and figure 3). However, this did not clearly occur in our results: instead, repetition obliterated the saccades effect. Thus, when these data were used to simulate the internal clock model, moderate correlations resulted.

The idea that suppression of brain activity can compress time perception was originally presented by Eagleman and Pariyadath as an energy model in time perception(12,19-21,27). The basic idea is that just before and during saccade execution (the perisaccadic interval) certain brain areas show reduced activity(37,40-42), and thus energy expenditure $(12,13,27)$. Since stimulus novelty (especially after a repeated stimulus) can increase neural activity / energy expenditure, it can (according to this theory) also dilate perceived time $(13,27)$ (see supplementary figure 1). A limitation of this model is that the causal link from energy metabolism to information processing is indirect, at best. However, taking this theory at face value, when both effects (repetition and saccade) occur simultaneously, the level of energy 
468

469

470

471

472

473

474

475

476

477

478

479

480

481

482

483

484

485

486

487

488

489

490

491

492

493

494

495

spent in the brain will be decreased by one factor (the saccade) and increased by other (novelty after repetition). Again, this can be represented mathematically as a combination of two energies (two coefficients) that are spent in different brain regions. Based on this assumption, behaviorally, we expect that one effect would cancel or weaken another effect. The correlation between outputs of the scalar model and empirical data was significant but R-value remained in the moderate range. Thus, in this case, scalar models of time perception (e.g., internal clock, and energy models) were partially able to describe the joint time distortion effect of repetition and saccades.

Since the scalar timing models showed results with moderate accuracy to justify the joint time distortion effects of saccade and repetition, a SDNs model was employed as an alternative method. This model explains state-dependent computations in neural network level presents subjective time $(7,8,43-45)$. Previous SDNs modeling studies have showed that artificial neural networks can simulate the behavioural responses in different timing conditions such as rhythm perception(8) and can encode time-varying sensory and motor patterns(46). This approach is similar to other artificial neural network studies that try to model a connectivity structure between incoming visual/auditory stimuli and behavioural responses. By this way, nontemporal perceptual classification approaches (e.g. feedforward neural networks) that are usually working based on Hebbian law, can be used to classify and predict subjective time(29). Consistent with the above studies, our, artificial neural network was able to fit the data in our study. However, such a network can fit almost anything, including a random shuffle of our data, i.e., it may have succeeded through overfitting. Thus, while this model was able to retroactively predict our data, the prediction was not specific.

A common element between scalar timing and SDN models is that they do not explicitly consider the concept of time in modern physics (time in Minkowski space). Objective time in these models is supposed to be the sequence of events, leading to subjective time perception. There is an interesting similarity between internal clock model and Newtonian absolute time, because both frameworks assumed an absolute generator that can create passage of time (Table 1). On the other hand, the SDN models assume moments can be coded via a 

nontemporal classification network and a Hebbian law is involved in learning and recognition of temporal patterns. However, based on modern physics theories each observer measures time as an intrinsic property from the environment, and this property can be changed based on information (entropy) and speed of both the environment and observer $(32,47)$. If we consider the brain as a physical observer, the same timing parameters should be hold in the brain and in the physical universe (Table 1). As time is characterized as a vector in Minkowski space, we used this concept in our last modeling approach. This vector model was originally suggested for time perception in long durations(30) but it can be proposed as a general time perception model for all intervals(31).

These time vectors can be defined by entropy and speeds in a physical system. In physiological terms, entropy can be conceptualized through different hypothesis. For example, order/disorder of states in dynamical functional connectivity patterns can change information entropy in functional brain networks and this alteration may compress or dilate perceived time(30). From a thermodynamical perspective, fluctuation of brain tissue temperature (as a thermodynamical system that exchanges heat with its surrounding) changes brain entropy and likewise may alter perceived time(31).

Our modeling results show that the vector model $(30,31)$ exhibited both sensitive and specific simulations of joint saccadic and repetition time distortion effects, i.e. in this model separate saccade and repetition result inputs predicted the combination of both effects. Furthermore, this model presented accurate results for both experiment (regardless of duration of intervals and type of trials). By representing perceptual effects as vectors and predicting the angles between these (Figure 3), this model was able to predict their interactions accurately and specifically. Conversely, in this model different time distortion effects can be considered as vectors in different directions that are added/subtracted to/from the original time vector (A detailed mathematical description of the specific vector interactions in our model can be found in Supplementary Discussion).

Overall, the results of vector timing model confirmed our hypothesis that subjective time has deep similarities to physical time and satisfied the first postulate of the special relativity 
524 theory(47). In this model, more information than magnitude (that is considered in scalar

525 models) is thought to be required to model dilation and compression of subjective time. Then,

526 subjective time is characterized by vector units of time with two properties: magnitude and

527 direction. When two time distortion effects are simultaneously interfered subjective time, two

528 vectors with different magnitudes and directions are added to subjective time. Presumably, this

529 information theory model is instantiated at the level of neural networks and cellular signals, but

530 at this time the biological mechanism is unknown.

531 In conclusion, we show here that global theories of time perception can be used to predict

532 interactions between seemingly disparate experimental phenomena and conversely, those such

533 interactions can help test between global theories. In particular, this study is the first to show

534 the interaction between saccadic time suppression and repetition time dilation, and that this

535 interaction follows the specific predictions of the vector model of time perception. In terms of

536 broader implication, this is the first empirical-theoretical investigation that directly shows subjective time can be represented by the same time parameter as that used in physics.

Table 1: Objective and subjective time and relation between them in different time perception

540 models.

\begin{tabular}{|l|l|l|l|}
\hline Model & Objective time & Subjective time & Timing rule \\
\hline model & Successive events & $\begin{array}{l}\text { Number of neural } \\
\text { pulses }\end{array}$ & $\begin{array}{l}\text { Counting of pulses in specific brain } \\
\text { regions can generate time. Same } \\
\text { mechanism is suggested in } \\
\text { Newtonian framework for physical } \\
\text { time. }\end{array}$ \\
\hline Energy model & - & Energy spent in the & $\begin{array}{l}\text { The value of energy in the brain (a } \\
\text { scalar parameter) can generate } \\
\text { time. There is no identity in the } \\
\text { physics. }\end{array}$ \\
& & &
\end{tabular}




\begin{tabular}{|c|c|c|c|}
\hline $\begin{array}{l}\text { State dependent } \\
\text { network model }\end{array}$ & Successive events & $\begin{array}{l}\text { Spatiotemporal } \\
\text { classification } \\
\text { networks }\end{array}$ & $\begin{array}{l}\text { Different states of brain networks } \\
\text { can present time. Hebbian law } \\
\text { supports this idea. }\end{array}$ \\
\hline Vector model & $\begin{array}{l}\text { Physical time in } \\
\text { Minkowski space }\end{array}$ & $\begin{array}{l}\text { Physical time in } \\
\text { Minkowski space }\end{array}$ & $\begin{array}{l}\text { Physical properties of brain (i.e. } \\
\text { entropy and speed) can measure } \\
\text { subjective time. } \\
\text { Same mechanism is suggested in } \\
\text { modern physics for physical time. }\end{array}$ \\
\hline
\end{tabular}

542 Table 2: the performance of different models to predict the behavioural results in four different

543 i.e., condition 1) one repetition in Fixation Task $\times$ Saccade Task, condition 2) two repetitions in

544 Fixation Task $\times$ Saccade Task, condition 3) one repetition in Retina-Matched Fixation Task $\times$

545 Saccade Task, and condition 4) two repetitions in Retina-Matched Fixation Task $\times$ Saccade Task.

546 The $R^{2}$ and $p$-value were obtained from the multiple linear regression analysis. RMSE was

547 calculated based on the Euclidean distance between output of the models and behavioral data.

\begin{tabular}{|l|l|l|l|}
\hline Dataset & Scalar model & Vector model & ANN model \\
\hline Original data & $\mathrm{R}=0.64, \mathrm{p}$-value $<0.01$ & $\mathrm{R}=0.86, \mathrm{p}$-value $<0.01$ & $\mathrm{R}=0.78, \mathrm{p}$-value $<0.01$ \\
& $\mathrm{RMSE}=0.56$ & $\mathrm{RMSE}=0.32$ & $\mathrm{RMSE}: 0.49$ \\
\hline Random & $\mathrm{R}=-0.13, \mathrm{p}$-value $=0.26$ & $\mathrm{R}=0.15, \mathrm{P}$-value $=0.22$ & $\mathrm{R}=0.57, \mathrm{p}$-value $<0.01$ \\
shuffled data & $\mathrm{RMSE}: 0.93$ & $\mathrm{RMSE}=0.61$ & $\mathrm{RMSE}: 0.54$ \\
\hline
\end{tabular}


a) Scalar models (internal clock model)

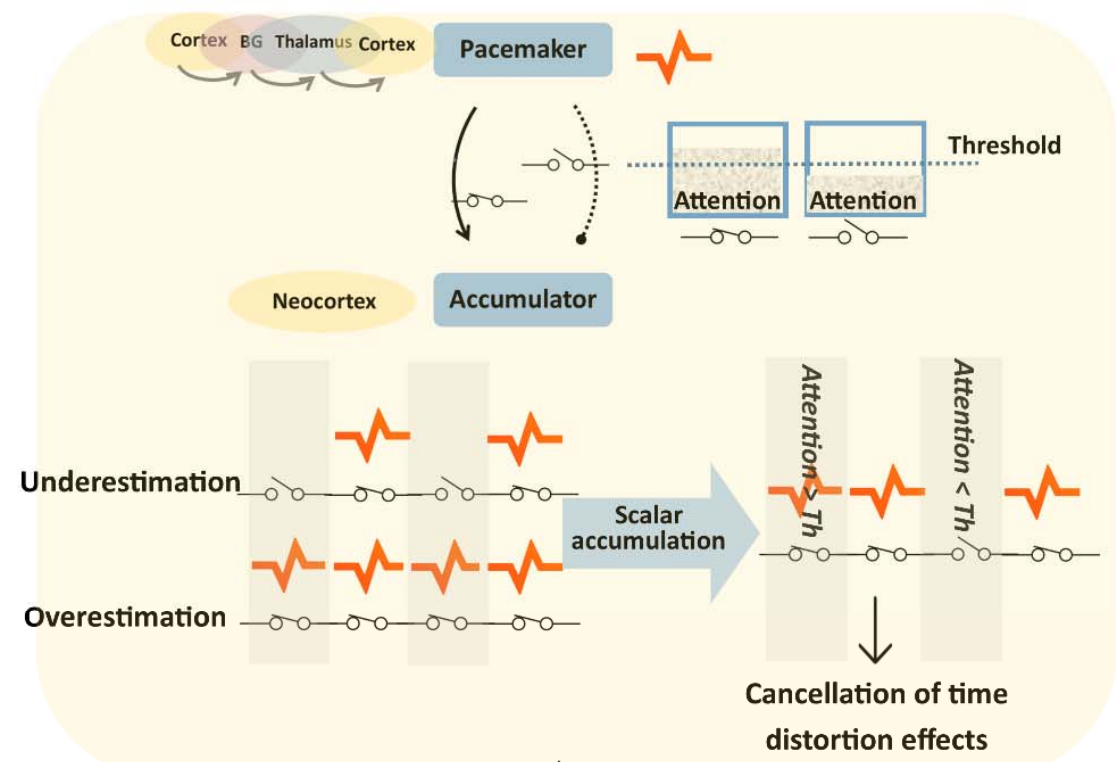

distortion effects b) State dependent network models

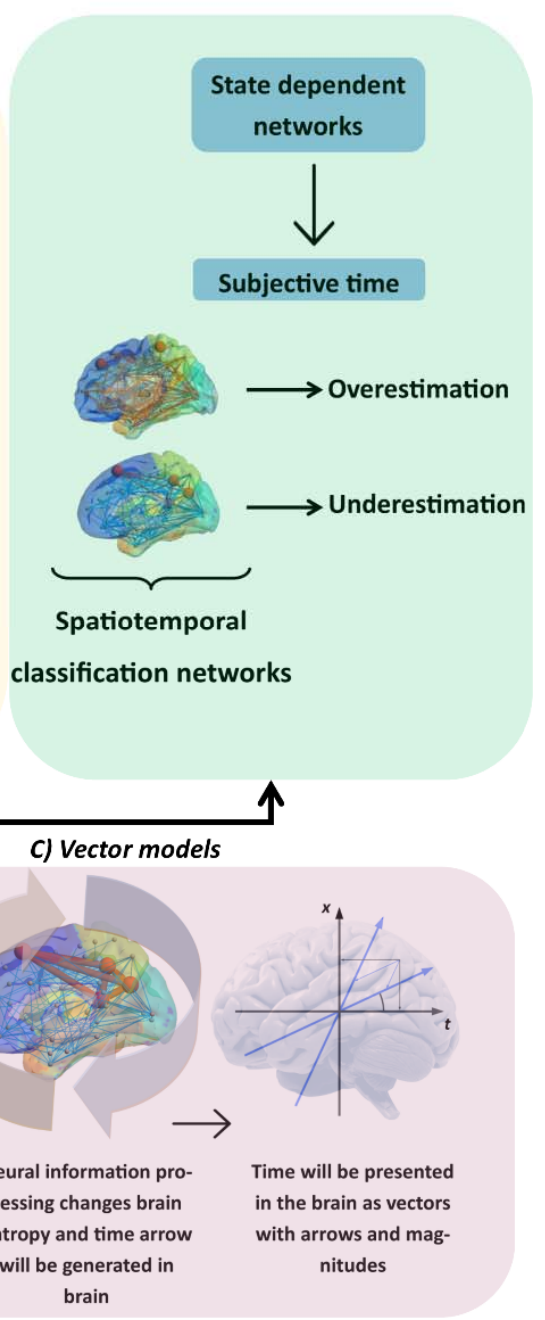

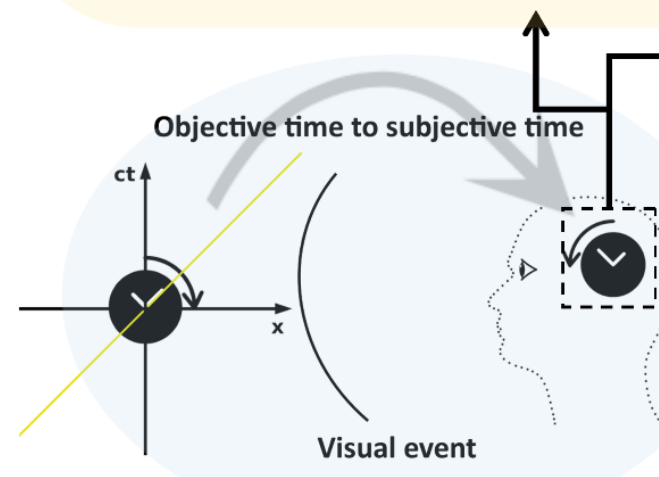

Visual event

\section{Figure 1:}

553 - How brain can process time. The objective time that is embedded in a visual event and

554 subjective time that is measured in the brain are characterized in two separate frameworks

555 (universe and brain). a) Scalar models) Internal clock model: In this model, an anatomical

556 pacemaker-accumulator structure is suggested to generate and collect scalar timing pulses. A

557 cortico-basal ganglion-thalamocortical pathway is suggested as pacemaker $(5,48,49)$ and

558 different cortical regions are suggested as accumulator $(5,25,48,50)$. In this model, attention is

559 assumed to connect the pacemaker to accumulator as a switch(3). Usually an attention 
560 threshold is considered, and the switch will be closed when the attention is higher than this

561 level. Otherwise, the switch is open, and accumulator cannot collect timing pulses. A higher

562 level of attention then leads to overestimation of time whereas lower attentional allocation will

563 lead to underestimation of time. When separate time distortion effects alter the level of

564 attention in different directions (one increases attention and the other decreases it) the 565 underestimation and overestimation effects cancel each other. b) State dependent network

566 models: In these models different states of neural networks reflect various time perception

567 states. The brain learns to distinguish long and short durations via Hebbian learning using a 568 spatiotemporal classification network instead of specialized temporal structures. c) Vector 569 model: A vector model explains the subjective and objective times are processed in the brain 570 and universe via same formulization. This model describes physical parameters (e.g., entropy 571 and speed that are involved in the measurement of objective time) are also reflected in 572 subjective time. For example, as the entropy of the universe is increased and objective time 573 passed, information processing in the brain increases the level of entropy in the brain and 574 subjective time passes. In this framework, brain and universe are considered as observer and 575 environment, respectively. Objective time can be measured in the brain based on physical time. 576 Both timing contexts can be formulated in Minkowski space as vectors (with arrows and 577 magnitudes). 
a)

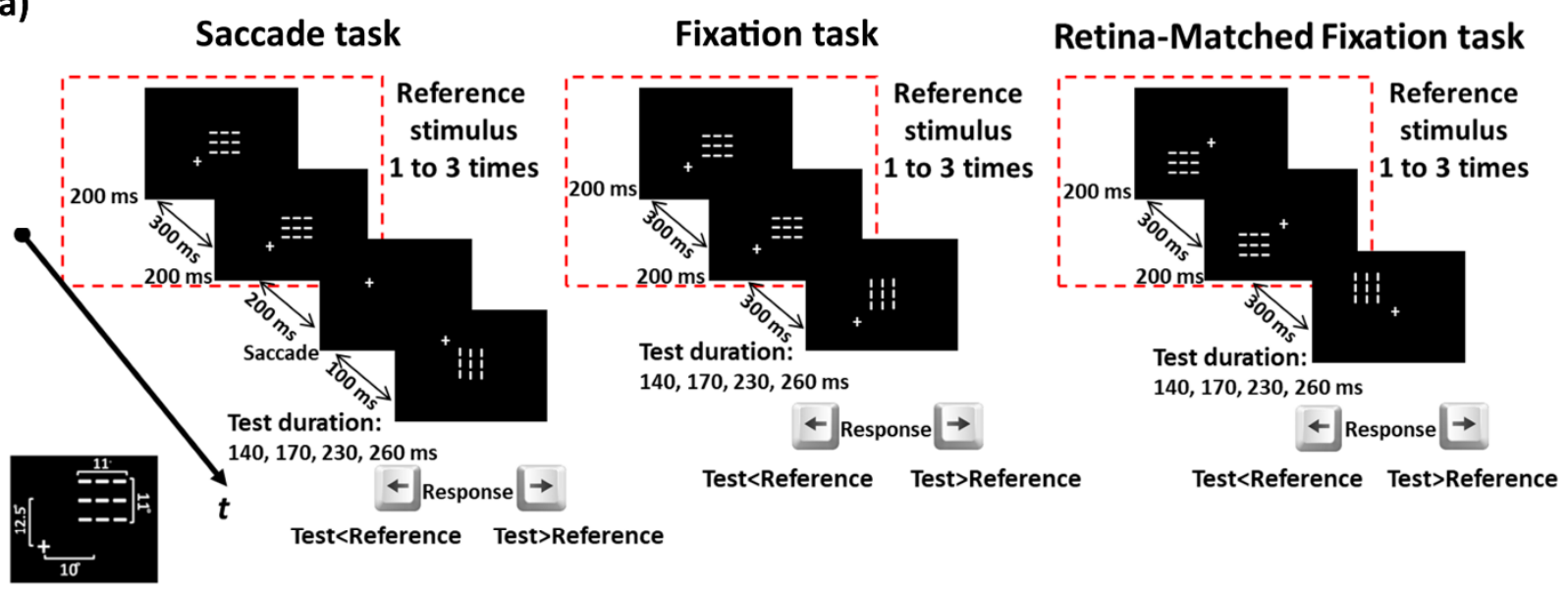

b)

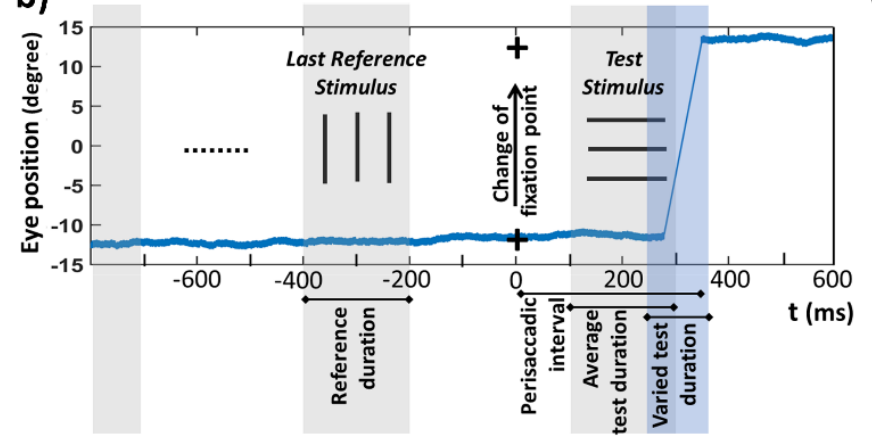

c)

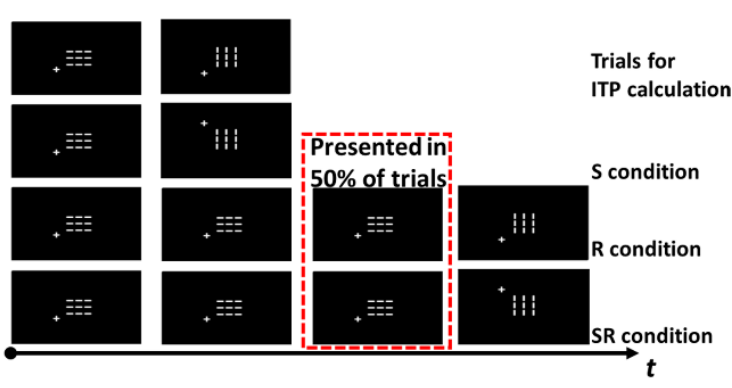

Figure 2: Behavioral task. a) Experimental design. We asked participants to judge the duration of a novel test stimulus (a horizontal or vertical grid) in comparison with the most recently presented reference stimulus (a grid with the opposite orientation. Participants fixed at one of the four corners relative to these stimuli. Three stimulus tasks (saccade, and two fixation controls) were presented in separate blocks, counterbalanced across. In each block, a series of 1-3 (randomized) $200 \mathrm{~ms}$ reference stimuli were presented at centre, followed by the test stimulus $(140,170,230$ or $260 \mathrm{~ms}$, randomized) at the same or 90 deg rotated orientation. After each trial, participants judged whether the test stimulus duration was shorter or longer than the reference stimulus. Participants always began fixating 8.3 degrees diagonally from one of the four corners of the reference stimulus location. In the Saccade Task, the fixation point shifted up or down to the opposite corner on the same horizontal side, $100 \mathrm{~ms}$ before test 592 presentation, eliciting a 25 degree vertical saccade during display of the test stimulus (and 593 causing the retinal location of the stimuli to shift). The Fixation Task was performed with fixed 594 gaze and stimuli locations whereas gaze was fixed in the Retina-Matched Fixation Task, but the 
595 location of the test stimulus was shifted to match the retinal locations of the Saccade Task. b) 596 Temporal evaluation and eye position in the Saccade Task. After presentation of the last 597 reference stimulus $(200 \mathrm{~ms})$, the location of fixation point was changed. Then, the test stimulus 598 was presented (after 100ms) and eye poison was changed (after saccade latency). Presentation 599 of the test stimulus (with variable durations) occurred during the perisaccadic interval. c) 600 Separate trials were used to study the time distortion effects of saccades (S), repetition (R) and 601 both saccades and repetition (SR). Control trials without saccades and repetition were used to 602 calculate individual time performances (ITPS) (see details in section 2-6-1). Statistical analysis 603 and modeling processes have been performed using this arrangement of trials. 
605

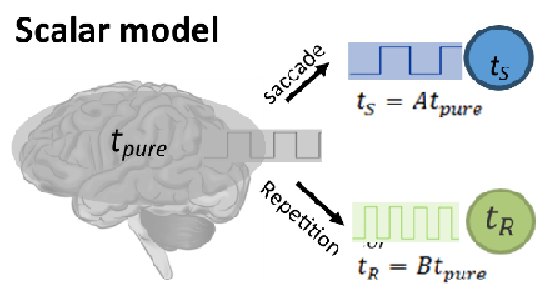

$$
\begin{array}{l|l}
t_{S} & \begin{array}{l}
t_{s}=A t_{\text {pure }} \rightarrow A=\frac{t_{s}}{t_{\text {pure }}}=I T P_{S} \\
t_{\omega_{2}}^{\text {Inputs }}
\end{array} \underbrace{\omega_{1}}_{t_{R}}
\end{array}
$$

Neural network model

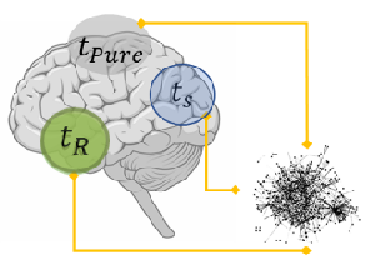

Vector model

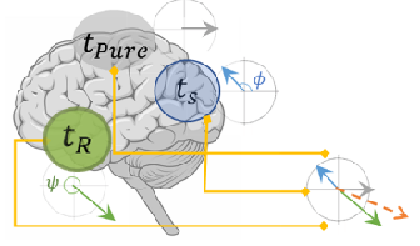

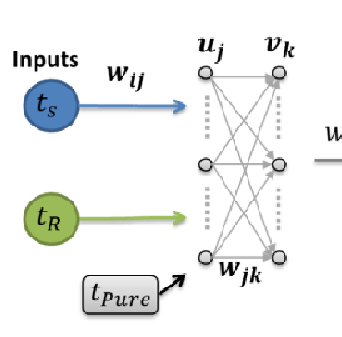

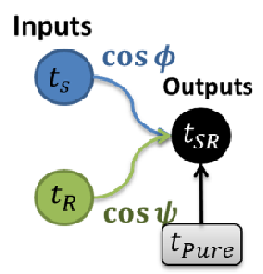

$t_{S R}=t_{s} \sum_{i, j, k} w_{i j} w_{j k} w_{k l} u_{j} v_{k}+t_{R} \sum_{i, j, k} w_{i j} w_{j k} w_{k l} u_{j} v_{k}+$ $t_{\text {pure }} \sum_{i, j, k} w_{i j} w_{j k} w_{k l} u_{j} v_{k}$

$\rightarrow t_{S R} / t_{\text {pure }}=\left(t_{S} / t_{\text {pure }}\right) \sum_{i, j, k} w_{i j} w_{j k} w_{k l} u_{j} v_{k}+$ $\left(t_{R} / t_{\text {pure }}\right) \sum_{i, j, k} w_{i j} w_{j k} w_{k l} u_{j} v_{k}+$

$\sum_{i, j, k} w_{i j} w_{j k} w_{k l} u_{j} v_{k}$

$\rightarrow I T P_{S R}=I T P_{S} \sum_{i, j, k} w_{i j} w_{j k} w_{k l} u_{j} v_{k}+$ $\operatorname{ITP} P_{R} \sum_{i, j, k} w_{i j} w_{i k} w_{k l} u_{j} v_{k}+\sum_{i, j, k} w_{i j} w_{j k} w_{k l} u_{j} v_{k}$

$t_{\text {Pure }}+t_{S} \cos \phi+t_{R} \cos \psi=t_{S R}$
$\rightarrow\left(t_{\text {Pure }}+t_{S} \cos \phi+t_{R} \cos \psi\right) / t_{\text {Pure }}=t_{\text {SR }} / t_{\text {Pure }}$
$\rightarrow 1+I T P_{S} \cos \phi+I T P_{R} \cos \psi=I T P_{S R}$

Figure 3: Three models have been shown schematically and mathematically. The

were defined as the percentage of trials that were correctly judged in the saccade, repetition, and saccade/repetition conditions respectively and ITP was defined based on Eq. 1. In all the models, inputs are the ITP in $S$ and $R$ conditions and output is ITP in SR condition. Above) In the linear scalar timing model, an internal clock generates clock pulses and distortion effects (saccade and repetition) change the rate of pulses. as two scalar parameters with an accumulative property. Mathematically (right side), in the scalar timing model one can assume that both time distortions effects (i.e. saccade $\left(t_{s}\right)$ and repetition $\left(t_{R}\right)$ ) can be added (by applying arbitrary coefficients or weights) and this scalar summation is equal to perceived time when both time distortion effects present (i.e. $t_{S R}$ ). Since the ITP is achieved from our experimental results, both sides of equation were divided by $t_{\text {pure }}$ (second line). Then based on eq. $1, t_{S, R, S R}$ were replaced by $I T P_{S, R, S R}$ (third line). All mathematical procedures in this approach are linear. Middle) In the nonlinear neural network model, time may be generated by state dependent networks. These networks were simulated by feedforward multilayer perceptrons. The synaptic weights are associated with a prior knowledge and based on these synaptic weights, network generates responses for new situations. Mathematically (right side), each time distortion effect 
$622\left(t_{s}\right.$ and $\left.t_{R}\right)$ multiplies by several synaptic weights between layers and adds to the time in absence

623 of distortion effects $\left(t_{\text {pure }}\right)$. This summation is equal to time distortion effects in the saccade and

624 repetition condition $\left(t_{S R}\right)$. As in the scalar timing model, both sides were dived by $t_{p u r e}$ and

625 according to eq. 1, 1, $t_{S, R, S R}$ were replaced by ITP $P_{S, R, S R}$ Below) In the linear vector timing model,

626 time units present as vectors in different angles. The angles may be defined by information

627 processing, entropy, or speed of neural processing. This model can present a computational

628 framework to explain how time units can be measured or integrated in the brain.

629 Mathematically (right side), time distortion effects $\left(t_{S}\right.$ and $\left.t_{R}\right)$ consider as two vectors and can

630 be added to the vector of pure time $\left(t_{\text {pure }}\right)$ via vector subtraction/addition operations. The

631 vector sum is performing by applying the cos of the angles between the vectors and this

632 summation is supposed to equal to perceived time in saccade and repetition condition ( $\left.t_{S R}\right)$ (the

633 first line). Then, both sides were divided by $t_{\text {pure }}$ to replace $t_{R, S}$ with $I T P_{R, S}$ (the second and third 634 rows).

635

636 
a)

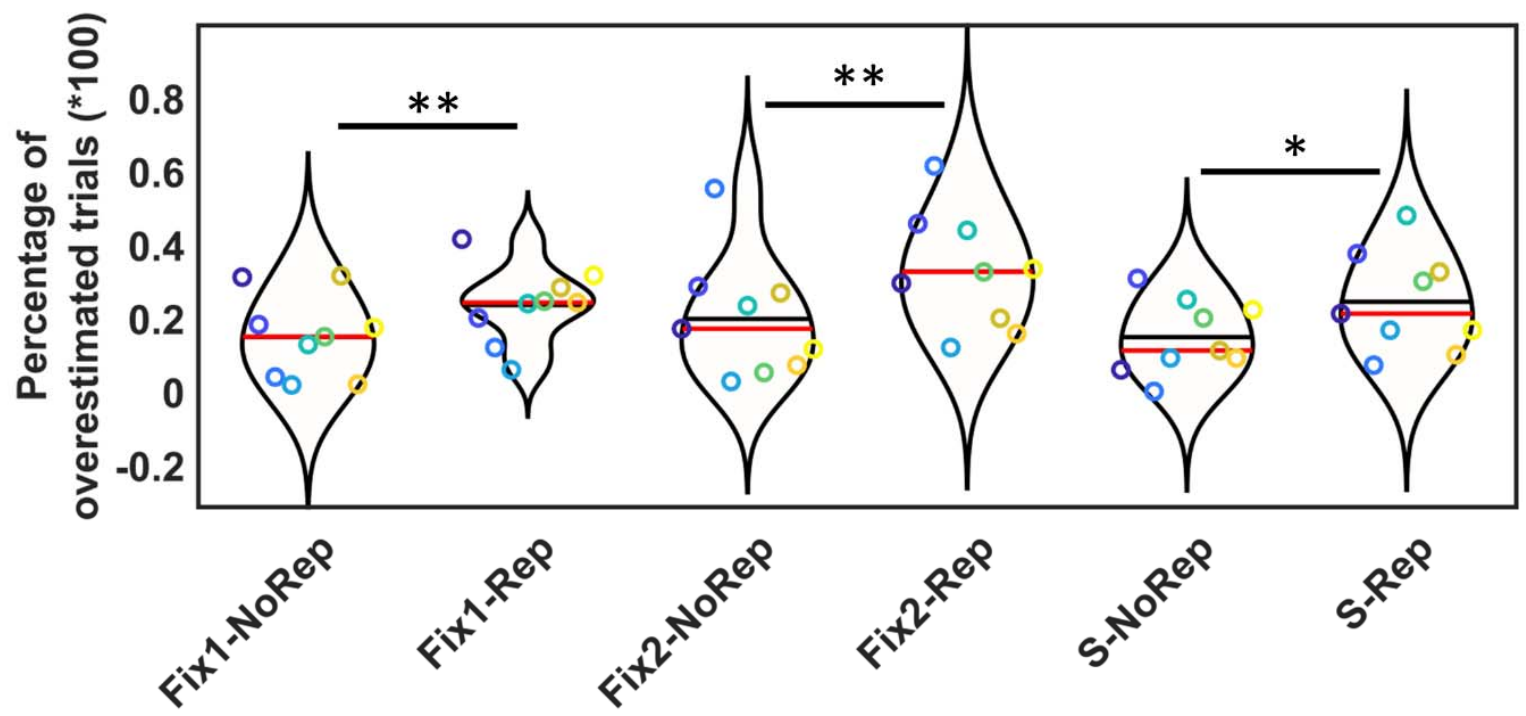

Conditions

b)

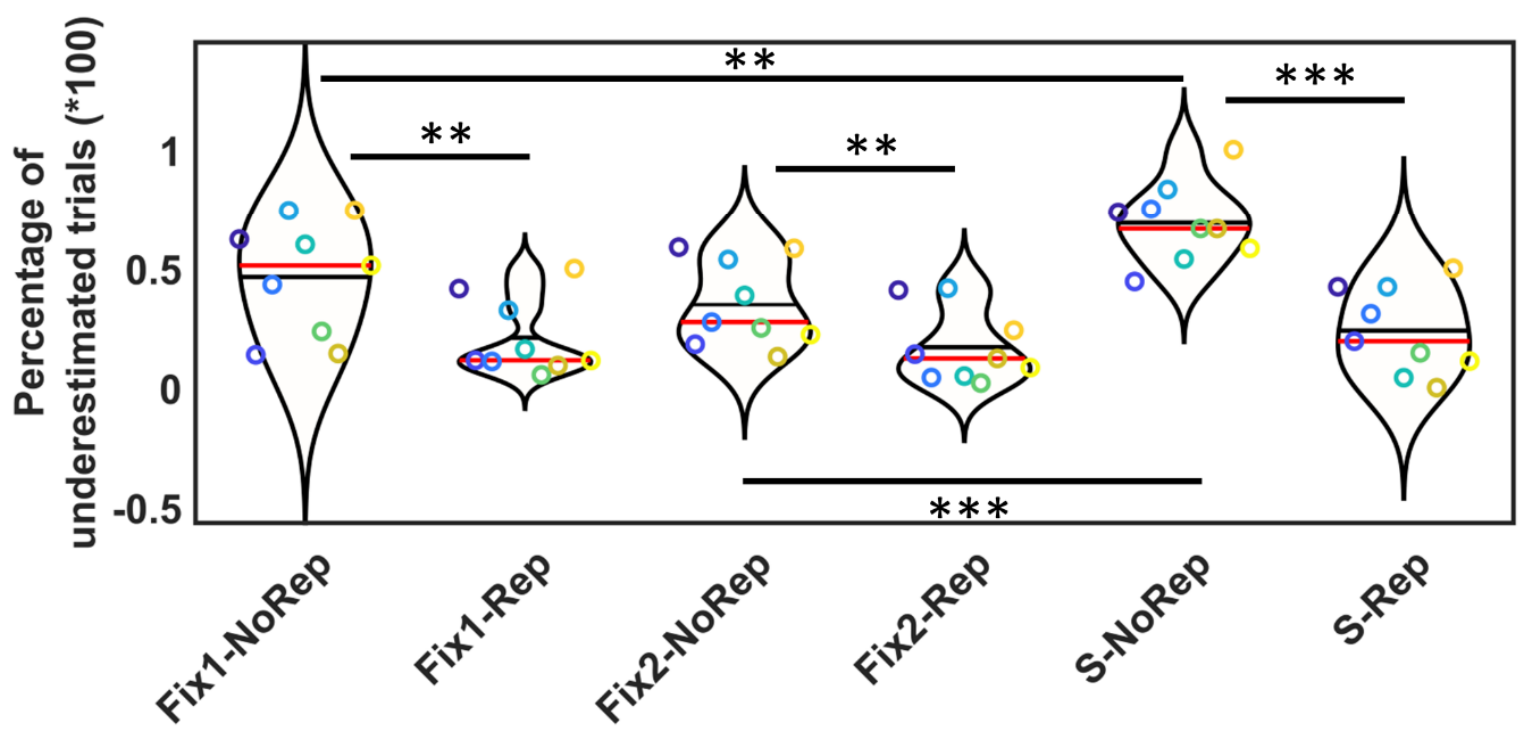

Conditions

Figure 4: Statistical results of the first experiment, showing significant results between different tasks and presentations of test stimulus. The distributions of responses were presented by

641 'violin plot' fits (where the horizontal axis shows different conditions, and the vertical axis 642 shows \% overestimated or underestimated trials). Individual responses were presented by color 
643 dots (responses of each participant can be tracked by a specific color and a specific vertical

644 location in each condition). One-star $(*)$ indicates $(p<0.05)$, two-stars $\left({ }^{*}\right)$ indicates $(p<0.01)$, 645 and three-stars $(* * *)$ shows $(p<0.001)$. Data are divided into incorrect responses for shorter 646 versus longer trials: a) Percentage of incorrect responses for shorter trials (overestimation of 647 time). Significant differences were observed between: Fix1-NoRep vs. Fix1Rep (t=-3.55, $648 p=0.0075)$, Fix2-NoRep vs. Fix2Rep ( $t=-3.79, p=0.0053)$, S-NoRep vs. S-Rep (t=-3.14, $p=0.013)$. b) 649 Percentage of incorrect responses for longer trials (underestimation of time). Significant 650 differences were observed between 1) repetition vs. no-repetition: Fix1-NoRep vs. Fix1-Rep $651(t=4.95, p=0.001)$, Fix2-NoRep vs. Fix2-Rep ( $t=4.62, p=0.002)$, S-NoRep vs. S-Rep ( $t=11.23$, $652 \mathrm{p}<0.001$ ), and tasks: S-NoRep vs. Fix1-NoRep ( $t=-3.63, \mathrm{p}=0.007)$, S-NoRep vs. F2-NoRep ( $\mathrm{t}=-7.49$, $653 p<0.001)$. 
a)

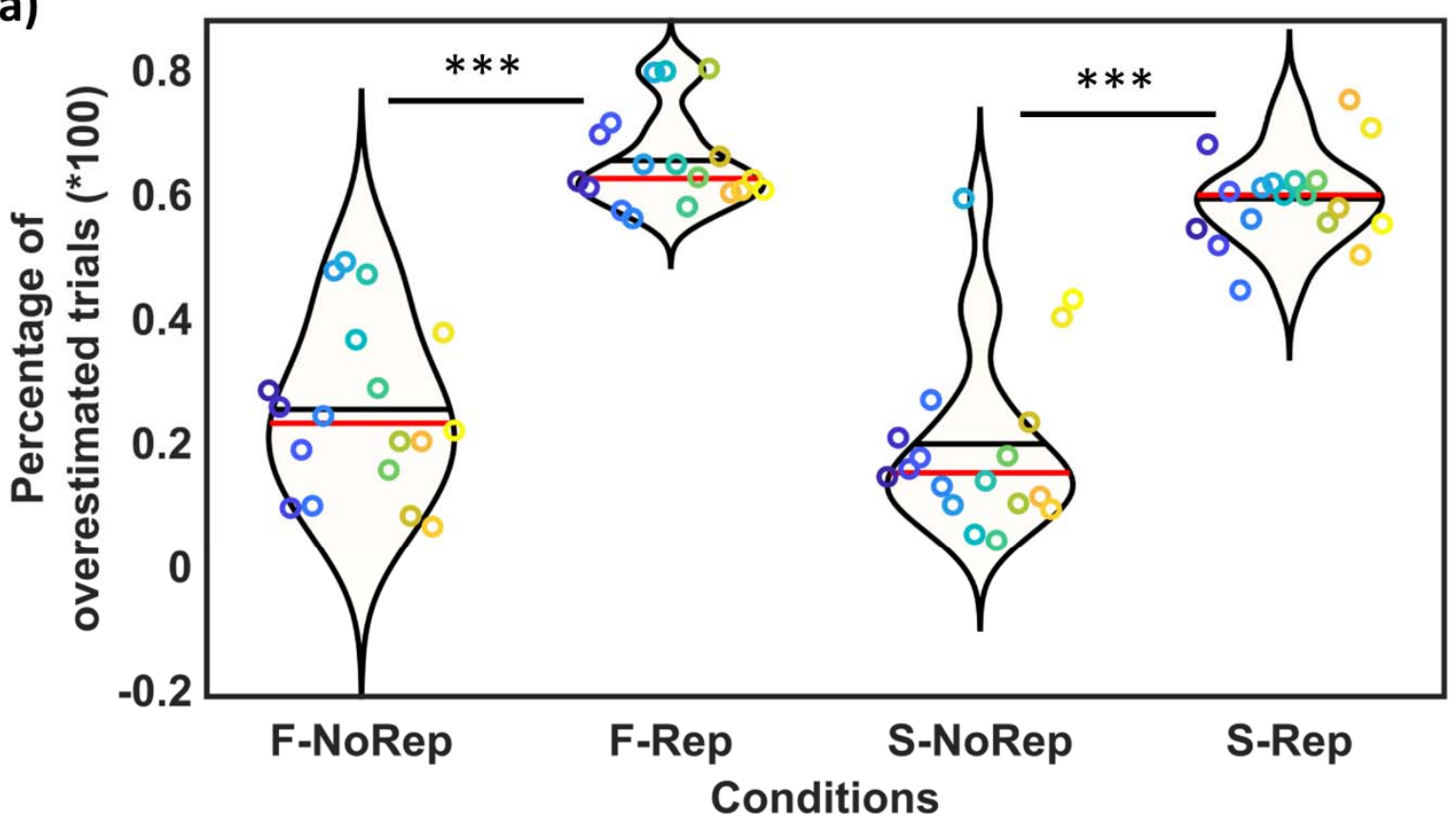

b)

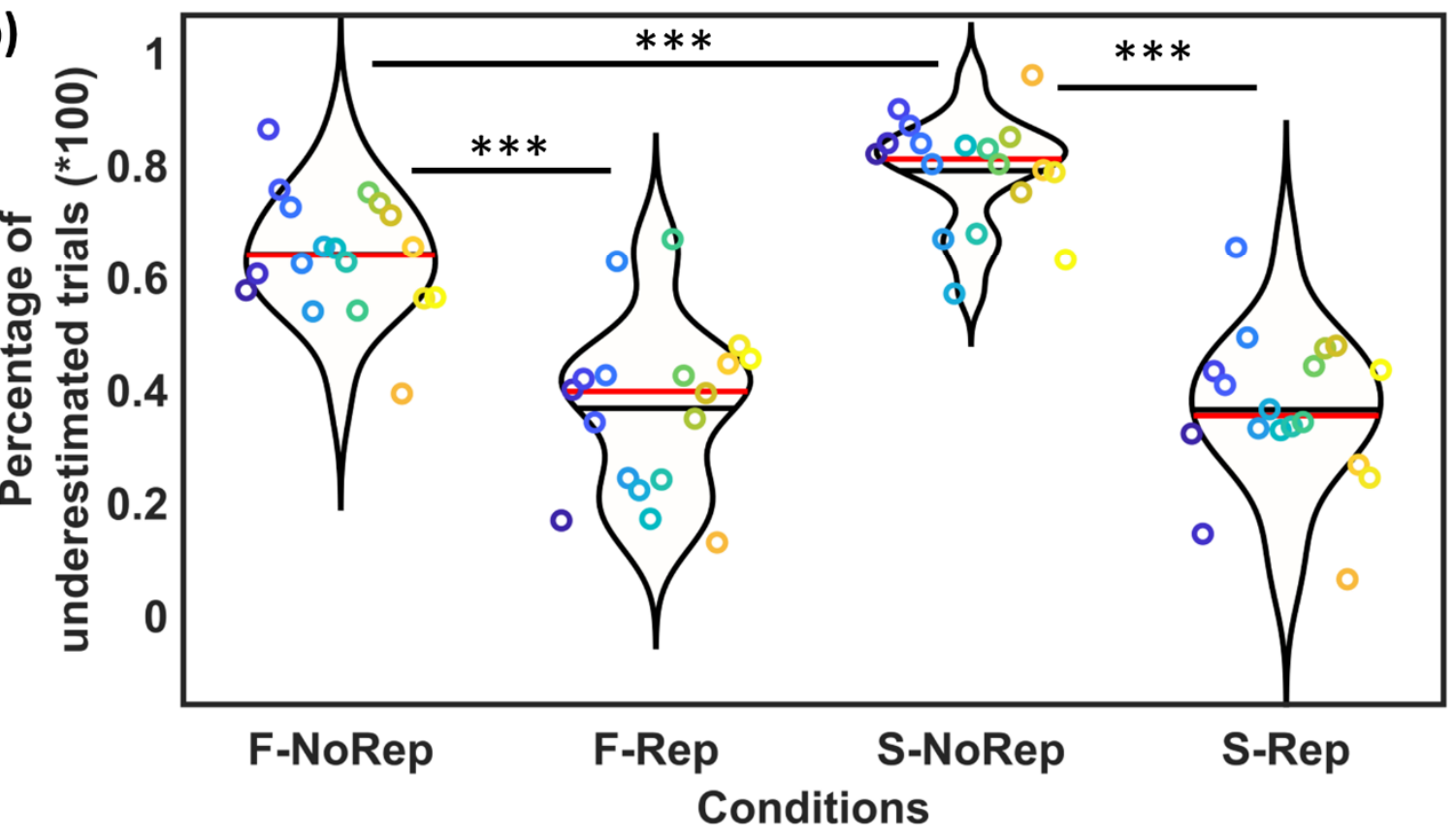

Figure 5: Statistical results of the first experiment, showing significant results between different tasks and presentations of test stimulus. The distributions of responses were presented by

657 'violin plot' fits (where the horizontal axis shows different conditions, and the vertical axis 658 shows \% overestimated or underestimated trials). Individual responses were presented by color 
659 dots (responses of each participant can be tracked by a specific color and a specific vertical

660 location in each condition). One-star $(*)$ indicates $(p<0.05)$, two-stars $\left({ }^{*}\right)$ indicates $(p<0.01)$, 661 and three-stars $(* * *)$ shows $(p<0.001)$. Data are divided into incorrect responses for shorter 662 versus longer trials: a) Percentage of incorrect responses for shorter trials (overestimation of 663 time). Significant differences were observed for repetition effect: F-NoRep vs. F-Rep (t=-12.40, $664 p<0.001)$, S-NoRep vs. S-Rep $(t(17)=10.71, p<0.001)$. b) Percentage of incorrect responses for 665 longer trials (underestimation of time). Significant differences were observed between 1) 666 repetition vs. no-repetition: F-NoRep vs. F-Rep $(t=6.93, p<0.001)$, S-NoRep vs. S-Rep $(t=10.02$, $667 p<0.001)$, and tasks: S-NoRep vs. F-NoRep $(t=-4.57, p<0.001)]$

668

669

670

671 
a)

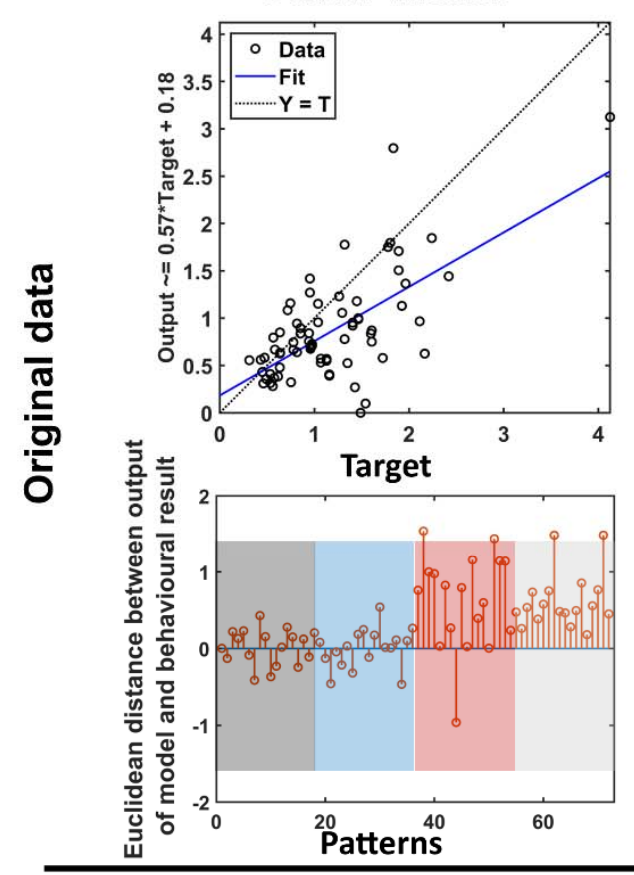

b)

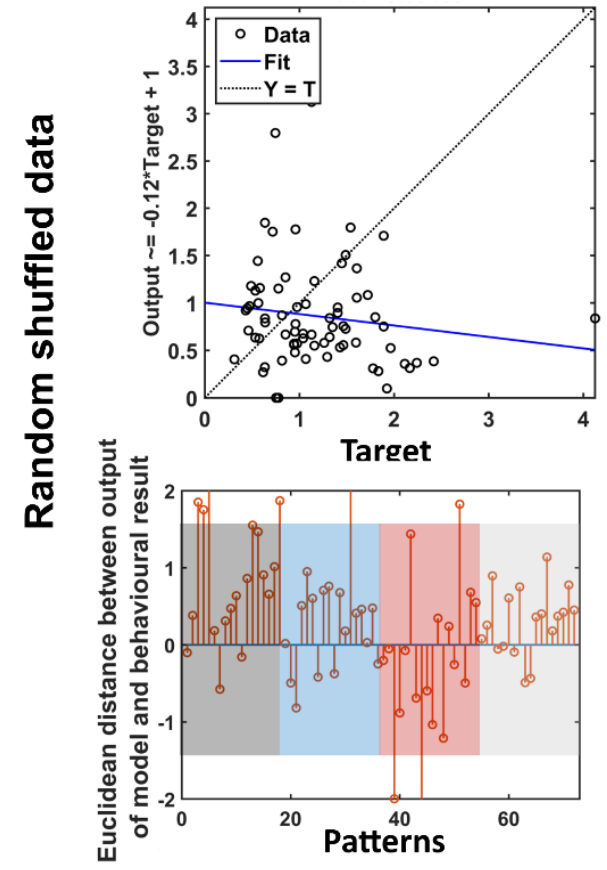

Neural Network Model
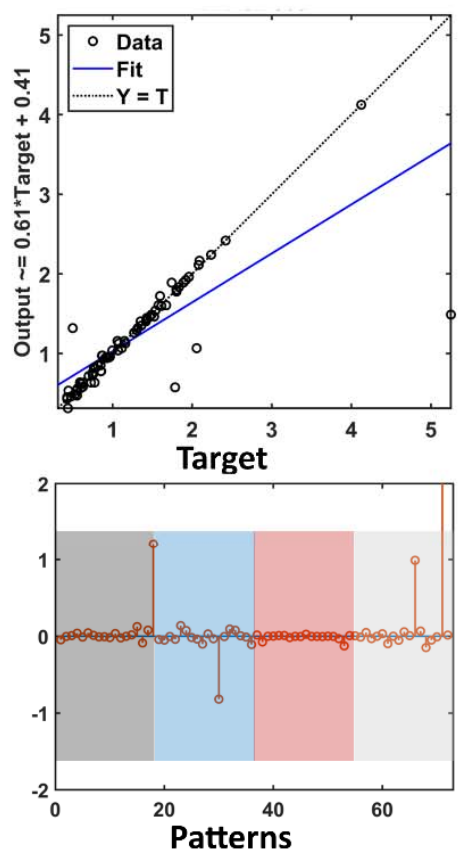

Neural Network Model
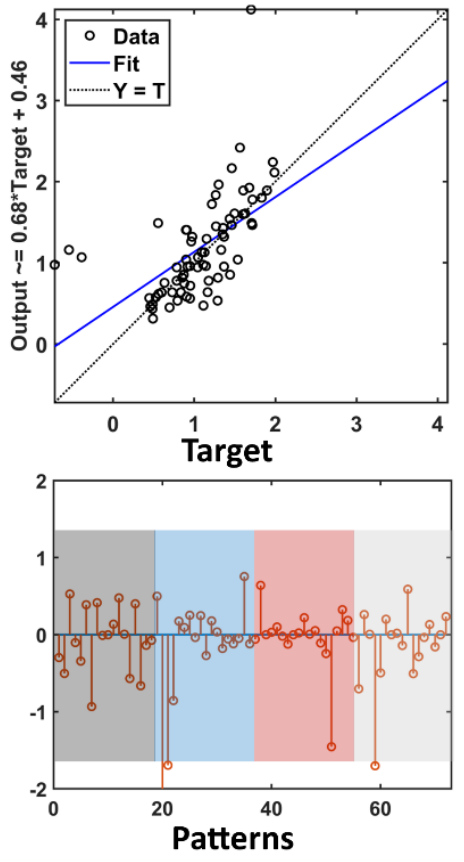

Vector Model
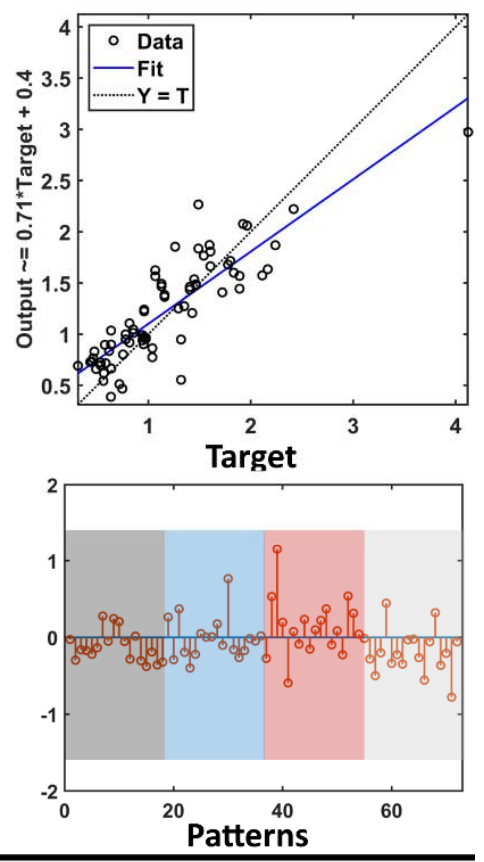

Vector Model
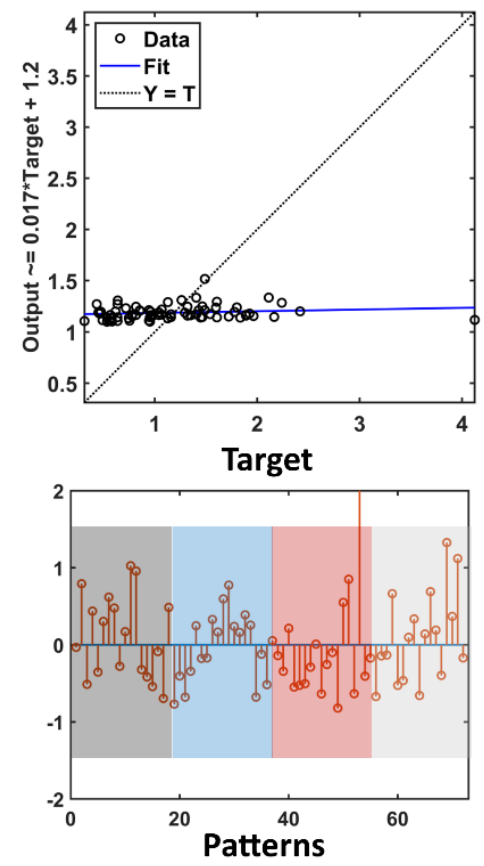

Figure 6: The correlations and residuals (Euclidean differences between output of models and

674 behavioural results) showing goodness of fit for each model, where high correlation and low 675 residuals signify good fit and higher residuals and lower correlation signify relatively poor fits. (a): correlation and residuals for fits to actual data. The residuals are sorted according to the 
677 experiments and trials: longer trials in second experiment (1 to 18; gray shadow), shorter trials 678 in second experiment (residuals19 to 36; blue shadow), longer trials in first experiment 679 (residuals 37 to 54; red shadow), shorter trials in first experiment (residuals 55 to 72 ; grey 680 shadow). (b): residuals between output of models and random shuffled patterns (across 681 participants and states) of behavioural results (outputs were assigned to irrelevant inputs (see 682 Methods for details). The colors are similar as the part (a). The Scalar Model (left column) yields 683 moderate fits for original data, the Neural Network Model (centre column) yields good fits, but 684 for both the original and shuffled data, whereas the Vector Model (right column) yields the best 685 fits (i.e., lowest residuals and high correlation) and only for the original data. 


\section{References}

687 1. Grondin S. From physical time to the first and second moments of psychological time. Psychol Bull. 2001;

2. Meck WH. Neuropsychology of timing and time perception. Brain Cogn. 2005;

3. GIBBON J, CHURCH RM, MECK WH. Scalar Timing in Memory. Ann N Y Acad Sci. 1984;

4. Ivry RB, Schlerf JE. Dedicated and intrinsic models of time perception. Trends in Cognitive Sciences. 2008.

5. Meck WH, Benson AM. Dissecting the brain's internal clock: How frontal-striatal circuitry keeps time and shifts attention. Brain Cogn. 2002;

6. Buonomano D V., Maass W. State-dependent computations: Spatiotemporal processing in cortical networks. Nature Reviews Neuroscience. 2009.

7. Karmarkar UR, Buonomano D V. Timing in the Absence of Clocks: Encoding Time in Neural Network States. Neuron. 2007;

8. Hardy NF, Goudar V, Romero-Sosa JL, Buonomano D V. A model of temporal scaling correctly predicts that motor timing improves with speed. Nat Commun [Internet]. 2018;9(1):1-14. Available from: http://dx.doi.org/10.1038/s41467-018-07161-6

9. Droit-Volet S, Meck WH. How emotions colour our perception of time. Trends Cogn Sci. 2007;

10. Wittmann M. The inner sense of time: How the brain creates a representation of duration. Nature Reviews Neuroscience. 2013.

11. Craig AD. Emotional moments across time: A possible neural basis for time perception in the anterior insula. Philosophical Transactions of the Royal Society B: Biological Sciences. 2009.

12. Eagleman DM. Distortions of time during rapid eye movements. Nat Neurosci. 
2005;8(7):850-1.

711

712

713

714

715

716

717

718

719

720

721

722

723

724

725

726

727

728

729

730

731

732

733

734

13. Eagleman DM. Human time perception and its illusions. Current Opinion in Neurobiology. 2008.

14. Morrone MC, Ross J, Burr D. Saccadic eye movements cause compression of time as well as space. Nat Neurosci. 2005;8(7):950-4.

15. Binda P, Cicchini GM, Burr DC, Morrone MC. Spatiotemporal Distortions of Visual Perception at the Time of Saccades. J Neurosci [Internet]. 2009;29(42):13147-57. Available from: http://www.jneurosci.org/cgi/doi/10.1523/JNEUROSCI.3723-09.2009

16. Penney TB, Cheng X, Leow YL, Bay AWY, Wu E, Herbst SK, et al. Saccades and subjective time in seconds range duration reproduction. Timing Time Percept. 2016;4(2):187-206.

17. Zimmermann E, Born S, Fink GR, Cavanagh P. Masking produces compression of space and time in the absence of eye movements. J Neurophysiol. 2014;

18. Balzarotti S, Cavaletti F, D'Aloia A, Colombo B, Cardani E, Ciceri MR, et al. The Editing Density of Moving Images Influences Viewers' Time Perception: The Mediating Role of Eye Movements. Cogn Sci [Internet]. 2021 Apr 1;45(4):e12969. Available from: https://doi.org/10.1111/cogs.12969

19. Sadeghi NG, Pariyadath V, Apte S, Eagleman DM, Cook EP. Neural correlates of subsecond time distortion in the middle temporal area of visual cortex. J Cogn Neurosci. 2011;

20. Pariyadath V, Eagleman D. The effect of predictability on subjective duration. PLoS One. 2007;

21. Pariyadath V, Eagleman DM. Subjective Duration Distortions Mirror Neural Repetition Suppression. PLoS One. 2012;

22. Yarrow K, Haggard P, Heal R, Brown P, Rothwell JC. Illusory perceptions of space and time preserve cross-saccadic perceptual continuity. Nature. 2001;414(6861):302-5. 
23. Terao M, Watanabe J, Yagi A, Nishida S. Reduction of stimulus visibility compresses apparent time intervals. Nat Neurosci. 2008;11(5):541-2.

24. Matthews WJ, Gheorghiu Al. Repetition, expectation, and the perception of time. Current Opinion in Behavioral Sciences. 2016.

25. Buhusi C V., Meck WH. Relativity theory and time perception: Single or multiple clocks? PLoS One. 2009;

26. Johnston A, Arnold DH, Nishida S. Spatially localized distortions of event time. Curr Biol. 2006;

27. Eagleman DM, Pariyadath V. Is subjective duration a signature of coding efficiency? Philosophical Transactions of the Royal Society B: Biological Sciences. 2009.

28. Mauk MD, Buonomano D V. THE NEURAL BASIS OF TEMPORAL PROCESSING. Annu Rev Neurosci. 2004;

29. Roseboom W, Fountas Z, Nikiforou K, Bhowmik D, Shanahan M, Seth AK. Activity in perceptual classification networks as a basis for human subjective time perception. Nat Commun. 2019;

30. Ghaderi AH, Moradkhani S, Haghighatfard A, Akrami F, Khayyer Z, Balcı F. Time estimation and beta segregation: An EEG study and graph theoretical approach. PLoS One. $2018 ; 13(4)$.

31. Ghaderi AH. Heat transfer, entropy and time perception: Toward finding a possible relation between subjective and objective time. Med Hypotheses. 2019;122:172-5.

32. Lebowitz JL. Boltzmann's Entropy and Time's Arrow. Phys Today. 1993;

33. Dace T. The arrow of time. Cosm Hist. 2018;

34. Wearden JH, Lejeune H. Scalar properties in human timing: Conformity and violations. Q J Exp Psychol. 2008; 
35. Burr DC, Ross J, Binda P, Morrone MC. Saccades Compress Space, Time, and Number. Space, Time Number Brain. 2011;14(12):175-86.

36. Grondin S. Duration discrimination of empty and filled intervals marked by auditory and visual signals. Percept Psychophys. 1993;

37. Prime SL, Vesia M, Crawford JD. Transcranial Magnetic Stimulation over Posterior Parietal Cortex Disrupts Transsaccadic Memory of Multiple Objects. J Neurosci. 2008;

38. Dunkley BT, Baltaretu B, Crawford JD. Trans-saccadic interactions in human parietal and occipital cortex during the retention and comparison of object orientation. Cortex. 2016;

39. Tse PU, Intriligator J, Rivest J, Cavanagh P. Attention and the subjective expansion of time. Percept Psychophys. 2004;

40. Thiele A, Henning P, Kubischik M, Hoffmann KP. Neural mechanisms of saccadic suppression. Science (80- ). 2002;

41. Bremmer F, Kubischik M, Hoffmann KP, Krekelberg B. Neural dynamics of saccadic suppression. J Neurosci. 2009;

42. Niemeier M, Crawford JD, Tweed DB. Optimal transsaccadic integration explains distorted spatial perception. Nature. 2003;

43. Buonomano D V., Maass W. State-dependent computations: Spatiotemporal processing in cortical networks. Nature Reviews Neuroscience. 2009.

44. Buonomano D V., Merzenich MM. Temporal information transformed into a spatial code by a neural network with realistic properties. Science (80- ). 1995;

45. Johnson HA, Goel A, Buonomano D V. Neural dynamics of in vitro cortical networks reflects experienced temporal patterns. Nat Neurosci. 2010;

46. Goudar V, Buonomano D V. Encoding sensory and motor patterns as time-invariant trajectories in recurrent neural networks. Elife. 2018; 
783 47. Einstein A, Lawson RW. Relativity: The special and the general theory. Relativity: The Special and the General Theory. 2012.

785

48. Pouthas V, George N, Poline JB, Pfeuty M, VandeMoorteele PF, Hugueville L, et al. Neural network involved in time perception: An fMRI study comparing long and short interval estimation. Hum Brain Mapp. 2005;

49. Matell MS, Meck WH. Cortico-striatal circuits and interval timing: Coincidence detection of oscillatory processes. Cognitive Brain Research. 2004.

50. Bueti D, Macaluso E. Physiological correlates of subjective time: Evidence for the temporal accumulator hypothesis. Neuroimage. 2011; 